\title{
Insights into the fracture mechanisms and strength of amorphous and nanocomposite carbon
}

\author{
M.G. Fyta, I.N. Remediakis, and P.C. Kelires \\ Physics Department, University of Crete, P.O. Box 2208, 710 03, Heraclion, Crete, Greece \\ D.A. Papaconstantopoulos \\ Department of Computational and Data Sciences, \\ George Mason University, Fairfax, VA 22030, USA
}

\begin{abstract}
Tight-binding molecular dynamics simulations shed light into the fracture mechanisms and the ideal strength of tetrahedral amorphous carbon and of nanocomposite carbon containing diamond crystallites, two of the hardest materials. It is found that fracture in the nanocomposites, under tensile or shear load, occurs inter-grain and so their ideal strength is similar to the pure amorphous phase. The onset of fracture takes place at weakly bonded $s p^{3}$ sites in the amorphous matrix. On the other hand, the nanodiamond inclusions significantly enhance the elastic moduli, which approach those of diamond.
\end{abstract}

The quest for new superhard materials, with hardness and thermal stability comparable to diamond, has generated intense research at both the fundamental and practical level. Carbon-based materials are considered to be the best candidates for extreme hardness. One of the most widely studied pure carbon materials is tetrahedral amorphous carbon $(t a-\mathrm{C})$, containing a high fraction of $s p^{3}$ hybrids, with many desirable diamondlike properties 1 ]. The nanohardness of $t a-\mathrm{C}$ with $85-90 \% s p^{3}$ bonding has been measured to range from $\sim 50-60 \mathrm{GPa}[2]$ to $\sim 80 \mathrm{GPa}[3]$, compared to $100 \mathrm{GPa}$ for diamond, which makes it one of the hardest materials.

Nanocomposite carbon is expected to further improve the mechanical properties of pure amorphous phases through the appropriate choice of nanoinclusions. Such a prototypical nanocomposite carbon material consists of diamond crystallites embedded in a $t a-\mathrm{C}$ matrix. The stability of this phase, denoted by $n-\mathrm{D} / a-\mathrm{C}$, was theoretically predicted by Fyta et al. [4]. Its synthesis and growth mechanisms have been explored by Lifshitz and co-workers, in both hydrogenated [5] and pure [6] dense $a$-C matrices.

Despite the importance of amorphous and nanocomposite carbon systems, several critical aspects of their elastic properties and strength remain elusive. The elastic moduli of $t a-\mathrm{C}$ and of "amorphous diamond" ( $a$-D), a hypothetical $100 \% s p^{3}$-bonded model, were calculated to be lower than diamond's 7]. This indicates that continuous random networks are softer than the equivalent crystal, despite containing the same bonds, but the cause is unclear. Even more, the deformation properties beyond the elastic regime of $t a-\mathrm{C}$, widely used in applications as a hard coating, and of the equivalent nanocomposite $n-\mathrm{D} / a-\mathrm{C}$ are not yet known.

Some of the fundamental issues requiring investigation are: (a) It is well known that diamond's strength is limited by the existence of easy-slip (cleavage) planes [8]. Such planes do not exist in $a$-D, or $t a$-C. Would this make the latter yield at higher stress than diamond and, if not, what is the cause of weakening? (b) The elucidation of fracture mechanisms in both $t a-\mathrm{C}$ and $n$ - $\mathrm{D} / a$-C. Especially, one asks whether fracture in $n$ - $\mathrm{D} / a$-C occurs inter- or intra-grain, and which are the weak links leading to fracture. (c) Ultimately, is there any enhancement of elastic moduli and strength of the nanocomposite $n$-D/a-C compared to the pure ta-C phase? Similar questions can be asked for any nanostructured material.

Here, we present the results of state-of-the-art tight-binding molecular dynamics (TBMD) simulations, which shed light into these fundamental issues. We study both the elastic properties and the ideal strength and fracture mechanisms in amorphous and nanostructured carbon. We find that fracture in the nanocomposites occurs inter-grain, in the embedding matrix, and so the ideal strength is similar to that of $t a$-C. However, their elastic moduli are significantly enhanced over $t a$-C. The onset of fracture takes place at weakly bonded $s p^{3}$ sites.

The TB method is well suited for the present problem. It is more accurate than empirical schemes because it provides a quantum mechanical description of the interactions. On the other hand, while less accurate than ab initio approaches, it yields greater statistical precision and allows the use of larger cells.

We use the TB method developed at the Naval Research Laboratory (NRL) 9]. This is a non-orthogonal model, using distance- and environment-dependent parameters for transferability between different structures. The $s p$ parameters for $\mathrm{C}$ were obtained by fitting to a first-principles database, which includes both the band structure and energies of the diamond, graphite, sc, bcc, and fcc configurations, as well as of the $\mathrm{C}_{2}$ dimer. The fitting of the band structure (as a test, the predicted band structure of $\mathrm{C}_{60}$ matches independent LDA calculations) provides additional robustness to the TB Hamiltonian. This is an important advantage of the present TB method, which enforces the transferability 
of these parameters to the amorphous state. The fitting was extended to large nearest-neighbor distances, up to 6.6 $\AA$, so the approach properly describes the quantum mechanical effects of bond breaking and microfracture. The calculated phonon frequencies and elastic constants of diamond are reasonably close to experimental values. The equilibrium bulk, shear, and Young's moduli and the density of diamond are calculated at 480 (443) GPa, 494 (476) $\mathrm{GPa}, 1300(1145) \mathrm{GPa}$, and $3.65(3.51) \mathrm{g} \mathrm{cm}^{-3}$, respectively, compared to the experimental values given in parentheses. For a review of the NRL/TB scheme, see Ref. [10].

We use supercells of 512 atoms with periodic boundary conditions (PBC). The $n$ - $\mathrm{D} / a-\mathrm{C}$ structures contain a spherical nanocrystal in the middle, surrounded by dense amorphous carbon. It is essential to use cells of this size, something not feasible with first-principles MD methods, in order to describe the nanoinclusions properly, with respect to realistic sizes and volume fractions. Smaller cells are not realistic and are inadequate.

The nanocomposite structures were generated by melting and subsequent quenching at constant volume, in the canonical $(N, V, T)$ ensemble, of a diamond structure, while keeping the atoms in the central spherical region frozen in their positions [4]. After quenching, producing amorphization of the surrounding matrix, the cells are fully relaxed with respect to atom positions and volume. The density and coordination of the matrix is controlled by the initial starting volume of the supercell. The pure $a-\mathrm{C}$ cells are also generated by quenching from the melt. In both cases, the liquid was prepared at $6000 \mathrm{~K}$, and typical quenching durations and rates used are $40 \mathrm{ps}$ and $300 \mathrm{~K} / \mathrm{ps}$, respectively. In addition, we examine the properties of the Wooten-Winer-Waire (WWW) model [1] of $a$-D, which serve as a benchmark for the calculated properties of $a-\mathrm{C}$ and $n-\mathrm{D} / a-\mathrm{C}$ networks. The WWW structure (512 atoms) is fully relaxed with the NRL/TBMD approach. All calculated properties are inferred at $0 \mathrm{~K}$.

Two of the TBMD-generated structures, at equilibrium, are shown in Fig. 1. Panel (a) portrays a typical nanocomposite network. Due to the PBC, this corresponds to a special case, where we have a homogeneous dispersion of crystallites of equal size in the matrix, at regularly ordered positions. The diamond nanocrystal has a diameter of $12.5 \AA$ and its volume fraction is $31 \%$. The density of the matrix $\rho_{a m}$ is $3 \mathrm{~g} \mathrm{~cm}^{-3}$, and its mean coordination $\bar{z}_{a m}$ is 3.8. Panel (b) shows an equivalent single-phase $t a-\mathrm{C}$ network with the same $s p^{3}$ content and density. The $s p^{2}$ sites are largely clustered. The network contains a considerable fraction of both three- and four-membered (3- $m$ and 4- $m$ ) rings, as computed using the shortest-path criterion of Franzblau [12]. Specifically, there are 20 3- $m$ and 38 $4-m$ rings. The vast majority of sites involved in such small rings are $s p^{3}, \sim 95 \%$. The average bond length in $3-m$ $(4-m)$ rings is $1.50(1.55) \AA$. These characteristics are in excellent agreement with what state-of-the-art $a b$ initio MD simulations predict [13]. For example, in a network of a similar density with 125 atoms, three 3-m and eleven 4- $m$ rings, composed of $s p^{3}$ atoms, with average bond lenghts of $1.5-1.6 \AA$ were found. This comparison shows that the present TB method treats accurately the strain energy of $s p^{3}$ sites in small rings. Also, the density-coordination $\left(s p^{3}\right.$ fraction) relation for the $a$-C networks is found to be linear, in agreement with experiment and recent TBMD simulations using a different TB Hamiltonian [14].

We begin our analysis by examining the elastic properties of the networks. As a representative quantity, we calculated their bulk modulus $B$ as a function of the $s p^{3}$ fraction in the cells, by fitting the energy versus volume curves with the Murnaghan equation of state. Fig. 2 shows the variation of $B$ for various nanocomposite structures and for single-phase $a-\mathrm{C}$. The values for diamond and $a-\mathrm{D}$ are shown for comparison. The moduli of $t a-\mathrm{C}$ networks $\left(s p^{3}\right.$ fraction $\geq 60 \%$ [4]) are quite high, and that of $a$-D reaches $\sim 90 \%$ of the diamond value, confirming previous calculations [7, 14]. Clearly, the moduli of $n$-D $/ a$-C networks are considerably higher than those of $a$-C. A mean increase over $10 \%$ is evident. The enhancement becomes stronger as the $s p^{3}$ fraction, or the density, increases. A similar effect is seen as the size, or volume fraction, of the nanocrystals increases. Impressively, beyond a certain point, $B$ 's are shown to exceed the $a$-D value and approach that of diamond. We conclude that the elastic response of the composite structures to hydrostatic deformation is controlled by the nanoinclusions.

We now turn to the calculations of the ideal strength [15, 16] of the materials under study. This is the maximum stress that a material can sustain under non-hydrostatic loads before becoming unstable and yielding to plastic deformation or fracture. In order to check the validity of the employed method, we calculated the ideal tensile and shear strengths for diamond. From the stress-strain curves (not shown), and in the case of tensile load, we found 209 (225) GPa for the $\langle 100\rangle$ direction, 130 (130) for the $\langle 110\rangle$, and 124 (95) GPa for the $\langle 111\rangle$ direction. In the case of shearing, we found 130 (93) GPa for the $\{111\}\langle 112\rangle$ slip system. Our results are in good agreement with the $a b$ initio results of Telling et al. [8], given in parentheses, and confirm that the $\{111\}$ plane is the easy-slip plane in diamond. The corresponding tensile strain at the maximum strength is $0.28,0.21$ and 0.15 for $\langle 100\rangle,\langle 110\rangle$ and $\langle 111\rangle$, respectively. The critical shear strain is 0.23 .

Having established the reliability of the method, we proceed to the study of our amorphous and nanocomposite structures. For the latter, we apply tensile load in the $\langle 111\rangle$ direction and shear load on the $\{111\}$ plane in the $\langle 112\rangle$ direction. The crystallographic directions are those of the nanodiamond region. In both cases, we choose the easy-slip plane of diamond, so that we can have a direct comparison to diamond, and examine the possibility that such planes 
play a significant role in nanocomposites. The a-C phases are highly isotropic, therefore all directions of tensile or shear load are equivalent.

The structures were strained in a series of incremental strains. At each step, atomic positions were fully relaxed with TBMD. The stress was extracted by differentiating the energy with respect to strain. The resulting stress-strain data points and fitted curves, for both tensile and shear load, are shown in Fig. 3. There are a number of important aspects of these results. The first is that all structures have significantly lower ideal strengths and critical strains than diamond, including the $a$-D model which is $100 \% s p^{3}$-bonded and has no planes of cleavage.

Obviously, the difference stems from the weaker character of the $\mathrm{C}-\mathrm{C}$ bond in the amorphous network. Note that while the modulus of $a$-D reaches $90 \%$ that of diamond, its strength is only about half. This indicates softer angular forces, due to dihedral disorder, which render the $s p^{3}$ hybrids more easily unstable under non-hydrostatic stresses at the bond-breaking regime, far from equilibrium. On the other hand, radial rigidity near equilibrium is not so weakened, giving rise to high elastic moduli. In the case of $t a-\mathrm{C}$, we have, in addition, the presence of $s p^{2}$ sites, which might contribute to the further decrease of strength, as it is evident in Fig. 3. We analyze these issues more deeply below.

Our most striking finding is that the ideal strength of $n-\mathrm{D} / a-\mathrm{C}$ is about equal to that of $t a-\mathrm{C}$, when having equivalent amorphous networks, for both tensile and shear loads. It indicates that the nanoinclusions do not actually contribute to the increase of strength, but that this is rather controlled by the other part of the composite structure, the amorphous matrix.

In order to understand this result, we examined the fracture mechanisms in our networks. For this purpose, we analyzed the microstructure just after the critical strain when bonds start to break. We consider a bond as being broken when its length has become longer than the first minimum in the pair distribution function of the network at equilibrium [17]. The so extracted cutoff distances are $1.90 \AA$, for the amorphous networks, and $1.75 \AA$ for the nanodiamond crystallite. (For $n-\mathrm{D} / a$-C, the partial functions in the two components were extracted.)

Fig. 4 shows ball and stick models of $t a-\mathrm{C}$ and nanocomposite carbon under critical tensile strain. Atoms that have lost at least one bond are distinguished, according to their hybridization, from atoms with no broken bonds. It is clear that bond breaking in the nanocomposite takes place in the amorphous matrix and not in the nanodiamond, i.e., fracture occurs inter-grain and not intra-grain. Only at the interface some originally elongated nanodiamond bonds are broken. (Stress in the nanodiamonds is mostly concentrated towards the interface [4].) This picture explains why the ideal strengths and corresponding strains of $n-\mathrm{D} / a-\mathrm{C}$ and $t a-\mathrm{C}$ are about equal. The fracture mechanisms in both materials are similar.

Interestingly, the vast majority of atoms involving broken bonds are $s p^{3}$ hybrids, in both the $n$ - $\mathrm{D} / a$-C and $t a-\mathrm{C}$ materials. This can be understood on the basis of the relative energetics of $s p^{3}$ and $s p^{2}$ hybrids in the amorphous phase. It has been previously shown [18] that the average atomic energies of $s p^{3}$ and $s p^{2}$ sites in $t a-\mathrm{C}$ are -6.8 and -7.1 $\mathrm{eV}$, respectively, with respect to atomic $\mathrm{C}$. This is a huge energy difference between the two hybrid states compared to the almost degenerate energies in diamond and graphite $(\sim-7.4 \mathrm{eV})$. Therefore, in the presence of critical strain at the onset of fracture, when bonds have to break, it will be energetically favorable to loose bonds belonging to $s p^{3}$ atoms in the amorphous region. It is clear from Fig. 4 that microfracture originates in $s p^{3}$-rich clusters.

Then, what is the role of $s p^{2}$ sites, and why the strength of $t a-\mathrm{C}$ is lower than that of the all $s p^{3}$-bonded $a$-D? A close inspection of the $t a-\mathrm{C}$ network in Fig. 4(a) shows that the reduction is not directly linked to $s p^{2}$ sites, as only few of them have broken bonds, but indirectly through their spatial association with $s p^{3}$ sites. The fracture region involves many clustered $s p^{2}$ sites, but the weak links are exactly those $s p^{3}$ sites in their immediate vicinity. This is because these $s p^{3}$ atoms are under mostly tensile stress [7], contrary to the majority of $s p^{3}$ sites which are under the more favorable compressive conditions, and so they have higher atomic energies [18]. The unstable sites under strain easily give up a bond to become $s p^{2}$. This is the favored hybrid state on the surfaces [19] ultimately formed by fracture, when the material breaks apart. The absence of $s p^{2}$ sites in the WWW model, acting as precursors for the destabilization of $s p^{3}$ sites, explains its higher strength compared to ta-C. That the $s p^{2}$ sites are successible to larger plastic deformations is evident in the case of the $50 \%-50 \%$-C cell, as shown in Fig. 3.

Finally, by extracting the yield stress $Y$ (defined as the stress where the strain departs $0.2 \%$ from linearity) and the Young's moduli $E$ from the stress-strain curves of Fig. 3, and by using the empirical relation of Tabor [20], $H / Y=0.07+0.6 \ln (E / Y)$, we estimated the hardness $H$ of our networks. We find $\sim 90$ GPa for $a$-D (WWW), $\sim 70 \mathrm{GPa}$ for $t a-\mathrm{C}$ and $n-\mathrm{D} / a-\mathrm{C}$, and $\sim 40 \mathrm{GPa}$ for $50 \%-50 \% a-\mathrm{C}$, compared to $120 \mathrm{GPa}$ for diamond with the present TB method. The value for $t a-\mathrm{C}$ is in accord with the experimentally reported values. The nanocomposites offer a clear advantage over $t a$-C, namely their superior elastic properties. They are also expected to have excellent thermal stability, similar to that shown by $t a-\mathrm{C}$, and relaxed intrinsic stress, as proposed elsewhere [4]. Experimentally, stress relief can be achieved by moderate thermal annealing, as for $\mathrm{ta}$-C. The combination of these properties make nanocomposite carbon materials very useful for MEMS/NEMS devices. 
We are grateful to Shay Lifshitz for providing us with a preprint of the paper in Ref. [6]. This work is supported by a grant from the EU and the Ministry of National Education and Religious Affairs of Greece through the action

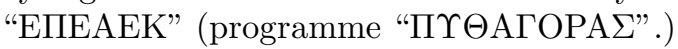

[1] For reviews see D.R. McKenzie, Rep. Prog. Phys. 59, 1611 (1996); J. Robertson, Mater. Sci. Eng. R 37, 129 (2002); S.R.P. Silva, in Handbook of Thin Film Materials, edited by H.S. Nalwa (Academic Press, New York, 2002), Vol. 4, p. 403.

[2] G.M. Pharr et al., Appl. Phys. Lett. 68, 779 (1996); X. Shi et al., Philos. Mag. B 76, 351 (1997); E. Martinez et al., Diamond Rel. Mater. 10, 145 (2001).

[3] T.A. Friedmann, K.F. McCarty, J.C. Barbour, M.P. Siegal, and D.C. Dibble, Appl. Phys. Lett.68, 1643 (1996).

[4] M.G. Fyta, I.N. Remediakis, and P.C. Kelires, Phys. Rev. B 67, 035423 (2003); M.G. Fyta, C. Mathioudakis, G. Kopidakis, and P.C. Kelires, Thin Solid Films 482, 56 (2005).

[5] Y. Lifshitz, T. Kohler, T. Frauenheim, I. Guzmann, A. Hoffman, R.Q. Zhang, X.T. Zhou, and S.T. Lee, Science 297, 1531 (2002).

[6] Y. Yao, M.Y. Liao, T. Kohler, T. Frauenheim, R.Q. Zhang, Z.G. Wang, Y. Lifshitz, and S.T. Lee, Phys. Rev. B 72, 035402 (2005).

[7] P.C. Kelires, Phys. Rev. Lett. 73, 2460 (1994).

[8] R.H. Telling, C.J. Pickard, M.C. Payne, and J.E. Field, Phys. Rev. Lett. 84, 5160 (2000).

[9] R.E. Cohen, M.J. Mehl, and D.A. Papaconstantopoulos, Phys. Rev. B 50, 14694 (1994); M.J. Mehl and D.A. Papaconstantopoulos, Phys. Rev. B 54, 4519 (1996); D.A. Papaconstantopoulos, M.J. Mehl, S.C. Erwin, and M.R. Pederson, Mater. Res. Soc. Symp. 491, 221 (1998).

[10] D.A. Papaconstantopoulos and M.J. Mehl, J. Phys: Cond. Matt. 15, R413 (2003).

[11] F. Wooten, K. Winer, and D. Weaire, Phys. Rev. Lett. 54, 1392 (1985); B.R. Djordjević, M.F. Thorpe, and F. Wooten, Phys. Rev. B 52, 5685 (1995).

[12] D.S. Franzblau, Phys. Rev. B 44, 4925 (1991).

[13] N.A. Marks D.R. McKenzie, B.A. Pailthorpe, M. Bernasconi, and M. Parrinello, Phys. Rev. Lett. 76, 768 (1996); D.G. McCulloch, D.R. McKenzie, and C.M. Goringe, Phys. Rev. B 61, 2349 (2000); N.A. Marks, N.C. Cooper, D.R. McKenzie, D.G. McCulloch, P. Bath, and S.P. Russo, Phys. Rev. B 65, 075411 (2002).

[14] C. Mathioudakis, G. Kopidakis, P.C. Kelires, C.Z. Wang, and K.M. Ho, Phys. Rev. B 70, 125202 (2004).

[15] Y. Zhang, H. Sun, and C. Chen, Phys. Rev. Lett. 93, 195504 (2004); ibid. 94, 145505 (2005).

[16] X. Blase, P. Gillet, A. San Miguel, and P. Melinon, Phys. Rev. Lett. 92, 215505 (2004).

[17] G. Galli, F. Gygi, and A. Catellani, Phys. Rev. Lett. 82, 3476 (1999).

[18] P.C. Kelires, Phys. Rev. Lett. 68, 1854 (1992); Phys. Rev. B 47, 1829 (1993).

[19] P.C. Kelires, J. Non-Cryst. Solids 227-230, 597 (1998); C.A. Davis, K.M. Knowles, and G.A.J. Amaratunga, Phys. Rev. Lett. 80, 3280 (1998).

[20] D. Tabor, Rev. Phys. Technol. 1, 145 (1970). 
(a)

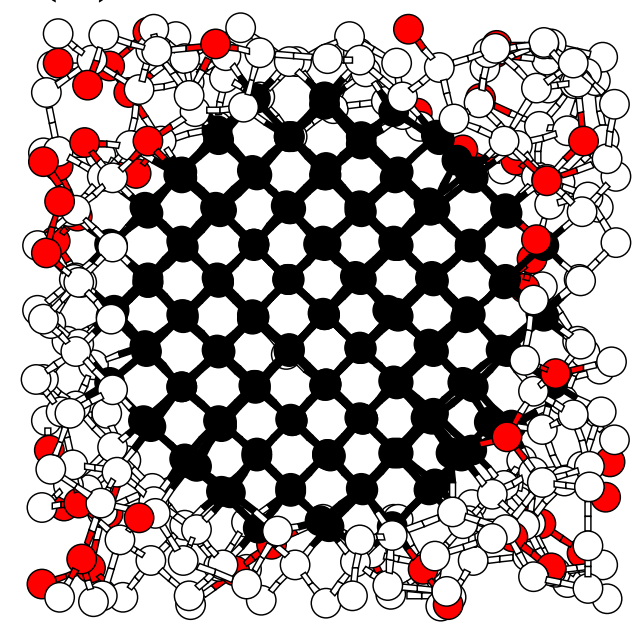

(b)

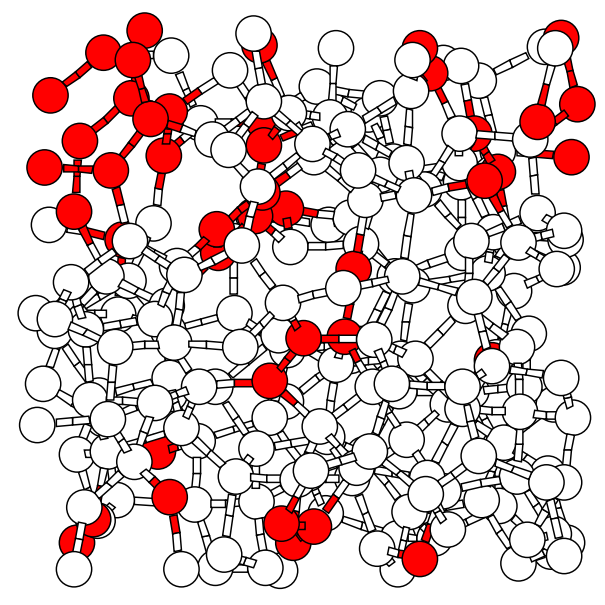

FIG. 1: (Color online) Ball-and-stick models for (a) a $n$-D/a-C network, and (b) a pure ta-C structure. Red (empty) spheres denote $s p^{2}\left(s p^{3}\right)$ sites. In (a), atoms belonging to the nanocrystal are represented by black spheres.

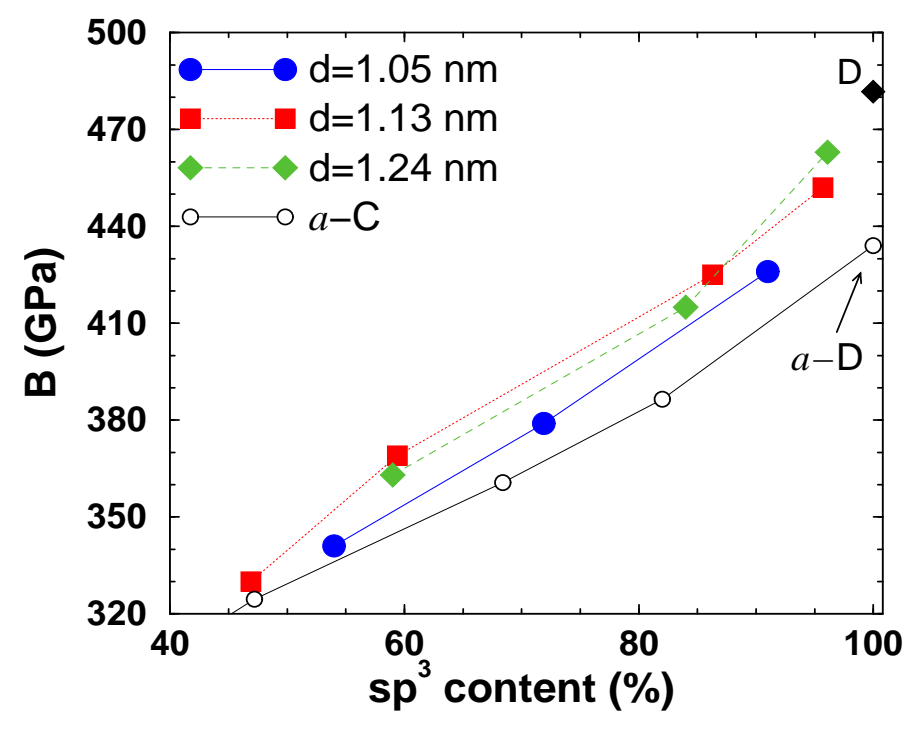

FIG. 2: (Color online) Bulk modulus as a function of $s p^{3}$ content for $a$-C and $n$-D $/ a$-C, with nanodiamonds of different diameter d. Calculations for bulk diamond (D) and $a$-D (the fully tetrahedral WWW model of a-C) are also shown for comparison. 

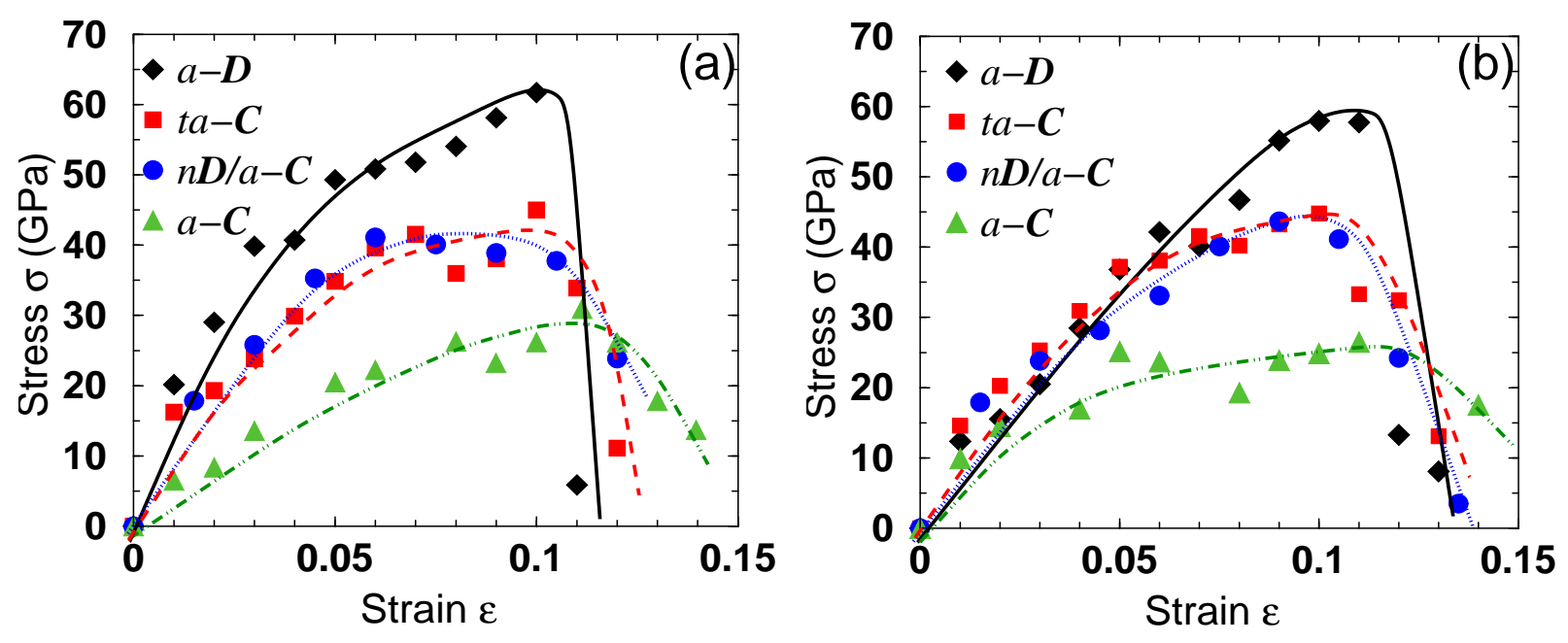

FIG. 3: (Color online) Stress versus strain curves for the various structures studied in this work. The $t a-\mathrm{C}$ and $n$-D/a-C contain $80 \% s p^{3}$ sites in the amorphous matrix; the $a$-C cell contains $50 \% s p^{3}$ sites. Lines are fits to the data points. (a) Tensile load in the (111) direction. (b) Shear load on the [111] plane in the (112) direction.

(a)

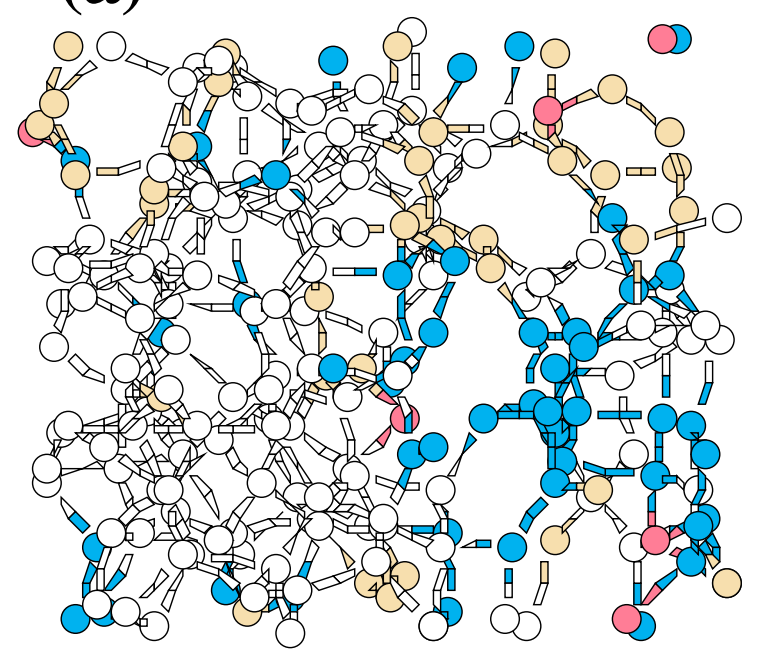

(b)

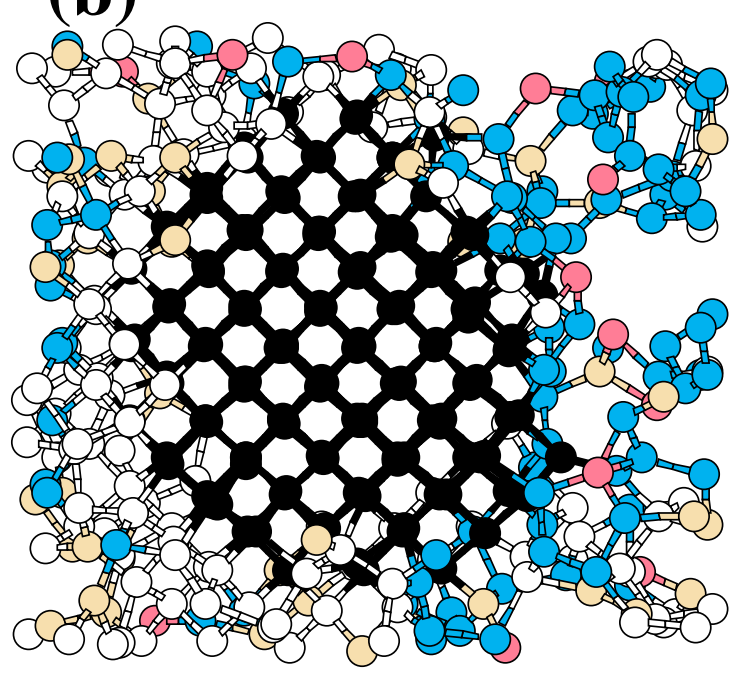

FIG. 4: (Color online) Ball-and-stick models for fracture in (a) single-phase $t a-\mathrm{C}$ and (b) nanocomposite $n$-D/a-C, of similar densities. Beige and empty spheres denote $s p^{2}$ and $s p^{3}$ sites with no broken bonds, respectively. Dark spheres show atoms of the nanodiamond. Pink and blue spheres denote broken $s p^{2}$ and $s p^{3}$ sites, which lost at least one bond, respectively. 